\title{
Ruptured intracranial dermoid cysts: a pictorial review
}

\author{
Jagoda Jacków ${ }^{1 B, D, E, F}$, George Tse ${ }^{2 B, E}$, Andrew Martinn ${ }^{2 B, E}$, Marek Sąsiadek ${ }^{10, E}$, Charles Romanowski2A,D,E \\ 'Department of General and Interventional Radiology and Neuroradiology, Wroclaw Medical University, Poland \\ ${ }^{2}$ Sheffield Teaching Hospital NHS Foundation Trust, Radiology, Royal Hallamshire Hospital, Sheffield, United Kingdom
}

\section{Abstract}

\begin{abstract}
Intracranial dermoid cysts are rare, benign, congenital, slow-growing cystic lesions. They are composed of mature squamous epithelium and can contain apocrine, eccrine, and sebaceous glands as well as other exodermal structures. Rupture of intracranial dermoid cysts is a relatively uncommon phenomenon but can cause more serious complications such as chemical meningitis, vasospasm, and cerebral infarction. Understanding of the appearance of both unruptured and ruptured dermoid cysts on computed tomography and MRI, especially awareness of existing low signal "blooming artefacts" on certain sequences, aids diagnosis and referral to the proper specialty for appropriate treatment.
\end{abstract}

Key words: dermatoid cyst, rupture, blooming artefacts, magnetic resonance imaging.

\section{Introduction}

Intracranial dermoid cysts are histologically benign cystic masses composed of mature squamous epithelium, and they can also contain apocrine, eccrine, and sebaceous glands as well as other exodermal structures such as hair follicles and teeth $[1,2]$. They account for approximately $0.5 \%$ of all primary intracranial tumours and are thought to be slightly more common in females [3]. Typically, dermoid cysts present in the first three decades of life $[3,4]$. They mostly occur in the midline suprasellar region, followed by the posterior fossa and also the spine. Malignant transformation into squamous cell carcinoma is extremely rare but has been described [2].

Dermoid cysts usually remain asymptomatic but can cause a variety of different symptoms, especially if they rupture. It is believed that dermoid cysts rupture secondarily to the production of hair and oils from internal dermal elements, which results in increasing pressure [2]. However, the rupture can be also preceded by a trauma [5]. Rupture of intracranial dermoid cysts is relatively uncommon, but the precise incidence of this phenomenon is not clearly known. In a single neurosurgical series of central nervous system tumour resections, ruptured dermoid cysts comprised only $0.18 \%$ of all tumours operated on during a 12-year period [5].

Clinical presentation critically depends on location. The most common symptoms are headache (32.6\%), followed by seizures $(26.5 \%)$, cerebral ischaemia (16.3\%), and aseptic meningitis (8.2\%) [6]. Specifically, patients with dermoid cysts located in posterior fossa can present with hydrocephalus caused by occlusion of the ventricular system [7]. However, in some cases cysts are also found incidentally. A rupture of the cyst presents with sudden headache, seizure, or with more serious complications such as chemical meningitis, vasospasm, and cerebral infarction.

\section{Radiological appearance of unruptured and ruptured intracranial dermoid cysts}

As already mentioned above, intracranial dermoid cysts are usually located in the midline, most commonly in the suprasellar cistern, followed by the posterior fossa. How-

\section{Correspondence address:}

Dr. Jagoda Jacków, Department of General and Interventional Radiology and Neuroradiology, Wroclaw Medical University, 213 Borowska St.,

50-556 Wroclaw, Poland, e-mail: jagodajackow@yahoo.pl

Authors' contribution:

A Study design · B Data collection · C Statistical analysis · D Data interpretation · E Manuscript preparation · F Literature search · G Funds collection 
Table 1. Radiological features of unruptured dermoid cysts

\begin{tabular}{|l|c|l|}
\hline \multicolumn{2}{|c|}{ Modality } & Radiological features \\
\hline \multirow{3}{*}{$\begin{array}{l}\text { Magnetic resonance } \\
\text { imaging }\end{array}$} & T1 & Typically high signal intensity with chemical shift artefacts \\
\cline { 2 - 3 } & T2/PD & Variable signal intensity ranging from low to high \\
\cline { 2 - 3 } & FLAIR & Heterogeneously hyperintense \\
\cline { 2 - 3 } & T2*GRE/SWI & Hypointense with "blooming artefacts" \\
\cline { 2 - 3 } & CE-T1 & Commonly do not enhance \\
\hline Computed tomography & $\begin{array}{l}\text { Hypodense lesion due to fat component } \\
\text { Commonly do not enhance }\end{array}$ \\
\hline
\end{tabular}

T1 - T1-weighted images, T2 - T2-weighted images, PD - proton density-weighted images, T2*GRE - T2*-weighted gradient echo images, SWI - susceptibility-weighted images, CE-T1 - contrast-enhanced T1-weighted images

Table 2. Radiological features of ruptured dermoid cysts

\begin{tabular}{|l|c|l|}
\hline \multicolumn{2}{|c|}{ Modality } & Radiological features \\
\hline $\begin{array}{l}\text { Magnetic resonance } \\
\text { imaging }\end{array}$ & T1 & $\begin{array}{l}\text { Typically high signal intensity within the lesion and in the subarachnoid space (fat droplets } \\
\text { within subarachnoid and/or subdural spaces or the ventricles) }\end{array}$ \\
\cline { 2 - 3 } & FLAIR & Subtle sulcal hyperintensity \\
\cline { 2 - 3 } & T2*GRE/SWI & Sulcal “bloom" \\
\cline { 2 - 3 } & CE-T1 & Leptomeningeal reaction and enhancement if complicated by chemical meningitis \\
\hline Computed tomography & $\begin{array}{l}\text { Hypodense fat droplets within subarachnoid and/or subdural spaces or the ventricles } \\
\text { Commonly do not enhance }\end{array}$ \\
\hline
\end{tabular}

T1 - T1-weighted images, T2 - T2-weighted images, PD - proton density-weighted images, T2*GRE - T2*-weighted gradient echo images, SWI - susceptibility-weighted images, CE-T1 - contrast-enhanced T1-weighted images

ever, they can also be found in the spinal canal. Diagnosis of the dermoid cysts and their rupture is based on imaging, including both computed tomography (CT) and magnetic resonance imaging (MRI). Tables 1 and 2 show typical radiological features of unruptured and ruptured dermoid cysts.

In CT the presentation of intracranial dermoid cyst is very characteristic (Figure 1A, 2A). They are typically well circumscribed, low attenuation lesions due to the fat components. However, there can be variable proportions of fat, hair, and epidermal debris. Calcifications can also be seen and have been reported to occur in $20 \%$ of cases [8].

In MRI dermoid cysts appears as high signal mass lesions on T1, fast spin echo T2, and FLAIR sequences (Figures $1 \mathrm{~B}-\mathrm{F})$. Chemical shift artefacts can also be observed, which occur at the fat/water interface in the frequency encoding directions. These artefacts arise due to the difference in resonance of hydrogen protons in fat and water as a result of their micromagnetic environment. The protons of fat resonate at a slightly lower frequency than those of water, and these are misregistered on the final image, creating the typical chemical shift artefact, as demonstrated in Figures 1, 2, and 3. High field-strength magnets are particularly susceptible to this artefact [8].

Rupture of dermoid cysts is reflected by the presence of the fat density/signal in the subarachnoid space or the ventricles (Figures 2 and 3). Some FLAIR sequences may be inherently "fat suppressed", which results in low signal from the fatty components of the ruptured dermoid cyst itself or the globules of fat in the subarachnoid space. Leptomeningeal reaction and enhancement can be observed if a ruptured dermoid cyst is complicated by chemical meningitis.

Other intracranial lesions and substances that should be considered in differential diagnosis can also demonstrate T1 high signal, e.g. gadolinium, haemoglobin degradation products, substances with high concentrations of protein or melanin (e.g. colloid cyst, craniopharyngioma, melanoma), and lesions containing mineral substances such as calcium, copper, and manganese (Figure 4) [9].

An important pitfall in imaging with $\mathrm{T} 2{ }^{\star}$ and susceptibility-weighted imaging (SWI) sequences is that there is an apparent "blooming artefact" within the dermoid and the sub-arachnoid fat droplets. These occur as areas of low signal, and in this circumstance can be easily confused as acute subarachnoid haemorrhage or haemosiderin staining instead of proper diagnosis of ruptured dermoid cyst. SWI performed with a spin-echo echoplanar imaging sequence with a long gradient echo train causes greater $\mathrm{T} 2{ }^{\star}$ weighting and as such is sensitive to haemosiderin susceptibility effects. This can be used as an adjunct to detect intracranial blood (Figures 4 and 5).

On diffusion-weighted imaging (DWI) the false assumption that an area of hypointensity on the B0 can, 

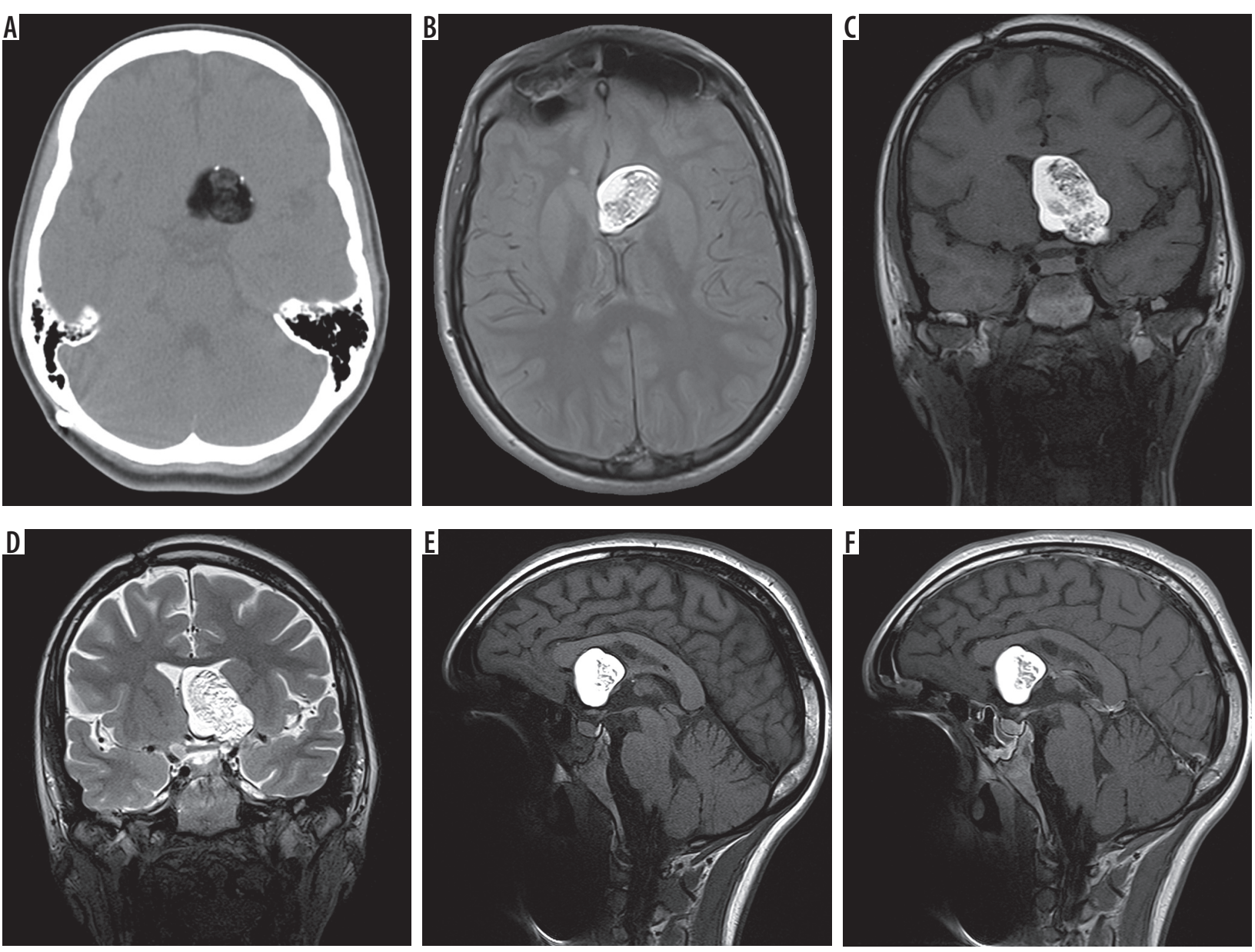

Figure 1. Unruptured dermoid cyst. A) Unenhanced axial computed tomography scan demonstrating a heterogenous predominantly fatty lesion with foci of peripheral calcification consistent with a dermoid cyst. B) Axial proton density-weighted images image confirms fatty nature of the lesion. C) Coronal T1-weighted image shows the lesion is suprasellar, and in the midline there is heterogenous internal structure with areas of high T1, representing fat, and isointense areas representing soft tissue/debris from dermoid epithelium. D) Coronal T2-weighted image; note the fat within the dermoid is higher in intensity than the adjacent cerebrospinal fluid. E-F) Pre and post-contrast sagittal T1-weighted images; there is no evidence of internal or rim enhancement to the dermoid cyst

however, lead to the incorrect interpretation that this represents a region of blood (whereas this is fat, and fat has no significant fluid to measure diffusion within). That is to say, fat has an intrinsic hypointensity on echoplanar imaging.

DWI is also an important tool in distinguishing epidermoid cysts from dermoid cysts. Dermoid cysts do not demonstrate restricted diffusion. The DWI signal is hyperintense to brain parenchyma, but there is no signal loss on the ADC map [10].

Knowledge of the appearances on CT and signal characteristics on MRI of dermoid cysts, especially in cases of their rupture, aid diagnosis and referral to the proper specialty to allow appropriate treatment, which is surgical resection of the lesion.

\section{Conclusions}

There is little literature on the incidence of rupture of dermoid; however, they can be a serious condition, which can cause not only headache and seizures but also cerebral infarction, hydrocephalus, chemical meningitis, and even death.

The lesion can be easily misdiagnosed due to the "blooming artefacts", with low signal seen on $\mathrm{T} 2^{*}$ and SWI being incorrectly interpreted as haemorrhage instead of fat. Understanding the physics behind $\mathrm{T}^{*}$ and SWI imaging and the "blooming artefact" phenomenon, as well as including other MRI sequences, is important in achieving the correct diagnosis.

\section{Conflict of interest}

The authors report no conflict of interest. 

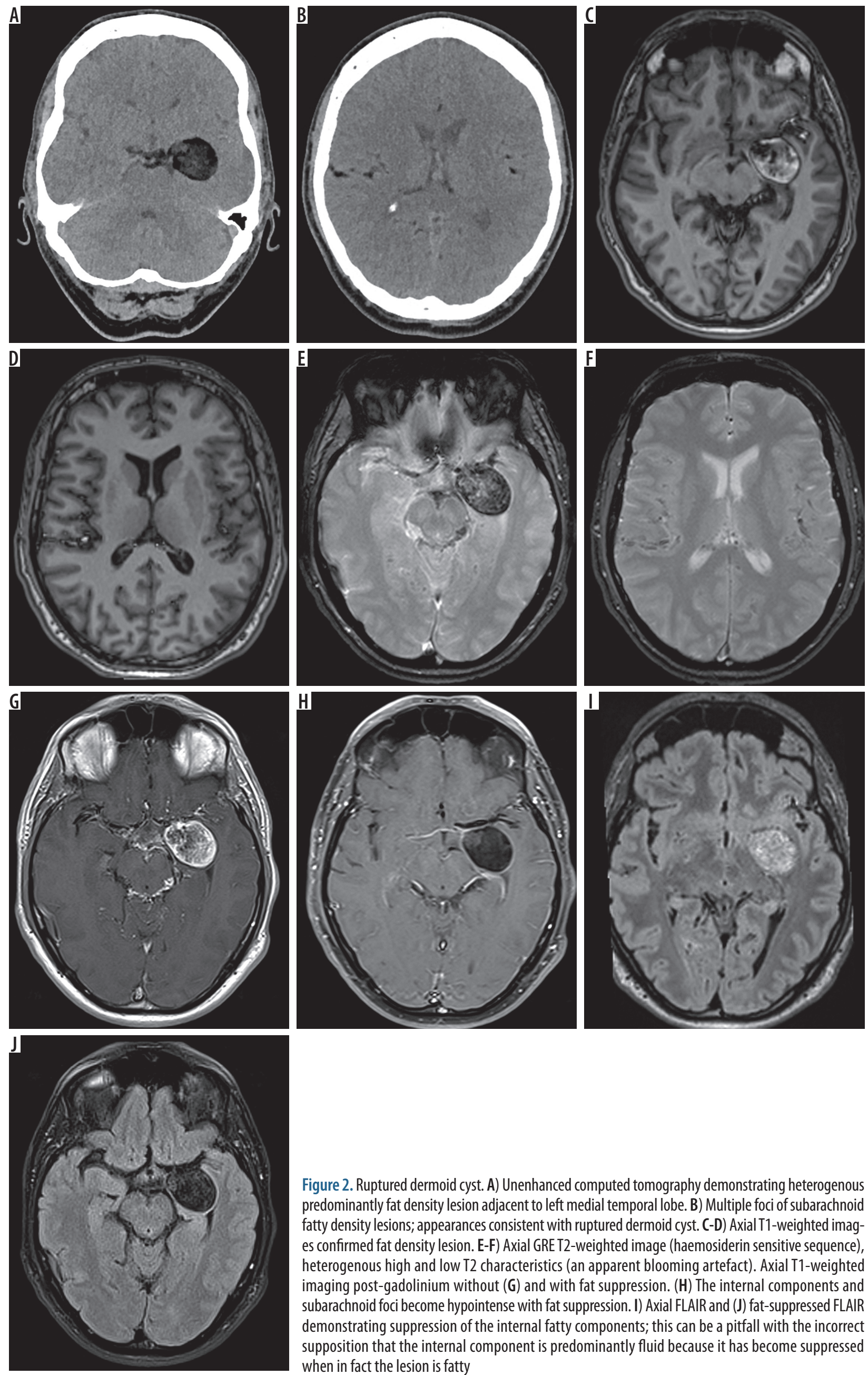

Figure 2. Ruptured dermoid cyst. A) Unenhanced computed tomography demonstrating heterogenous predominantly fat density lesion adjacent to left medial temporal lobe. B) Multiple foci of subarachnoid fatty density lesions; appearances consistent with ruptured dermoid cyst. (-D) Axial T1-weighted images confirmed fat density lesion. E-F) Axial GRE T2-weighted image (haemosiderin sensitive sequence), heterogenous high and low T2 characteristics (an apparent blooming artefact). Axial T1-weighted imaging post-gadolinium without $(\mathbf{G})$ and with fat suppression. $(\mathrm{H})$ The internal components and subarachnoid foci become hypointense with fat suppression. I) Axial FLAIR and (J) fat-suppressed FLAIR demonstrating suppression of the internal fatty components; this can be a pitfall with the incorrect supposition that the internal component is predominantly fluid because it has become suppressed when in fact the lesion is fatty 

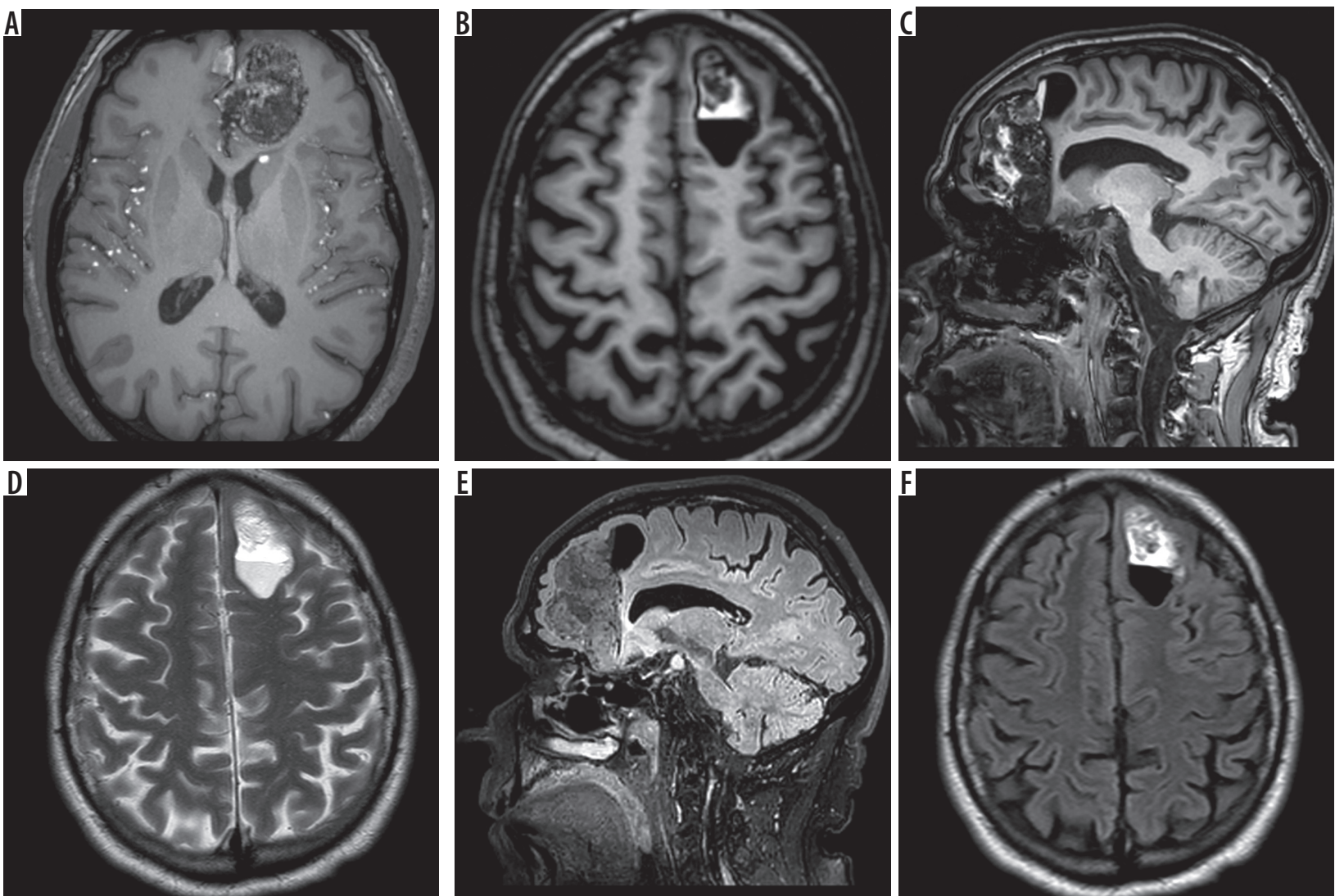

Figure 3. Ruptured dermoid cyst. A) Axial T1-weighted image demonstrating a ruptured left frontal dermoid with diffuse subarachnoid fat. B) Axial T1-weighted image demonstrating a well-defined level in the middle of the left frontal lesion. The anterior part has a heterogenous component and the posterior part is homogenously hypointense, consistent with a fat fluid level. C) Sagittal T1-weighted image the fluid component lies posterior to the larger fatty component obeying the normal laws of gravity with the patient supine in the magnetic resonance imaging scanner. D) Axial T2-weighted image again demonstrating the fat fluid level; note that the fat is significantly brighter, and the posterior fluid level is similar in intensity to the cerebrospinal fluid space signal. E) Sagittal reconstruction from fat suppressed 3D FLAIR demonstrates suppression of the posterior fluid component, but also notice the fat suppression, which does not become as hypointense as the nulled fluid. F) Axial FLAIR suppression of posterior fluid component with no change in the anterior fat component
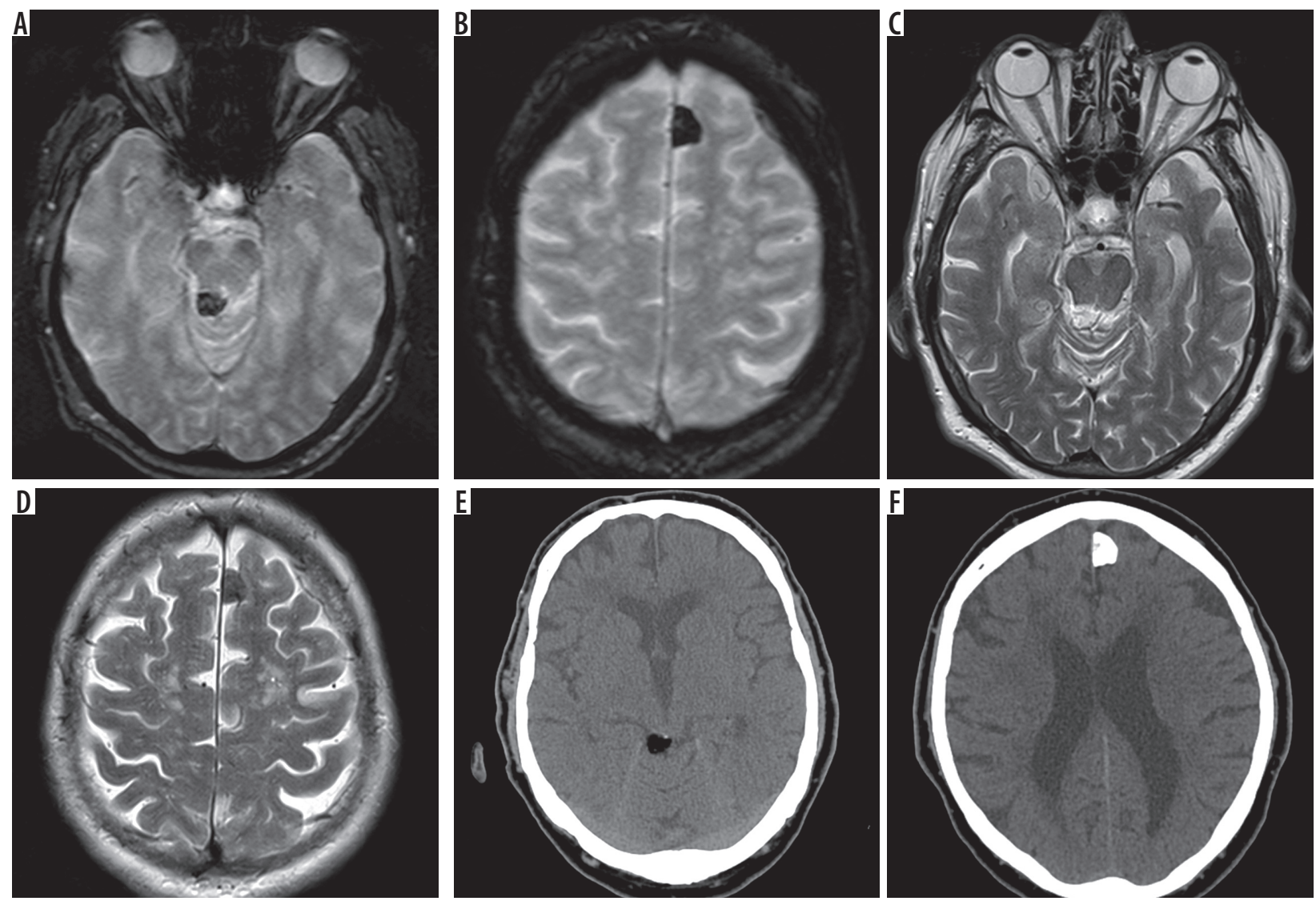

Figure 4. Differentiation of dermoid cysts. Pitfalls of susceptibility imaging. A-B) Axial GRE T2-weighted image (haemosiderin sensitive sequence), demonstrating a focal area of hypointensity in the left parafalcine position and in the quadrigeminal cistern. (-D) Axial T2-weighted image shows the quadrigeminal cistern lesion is heterogeneously hyperintense, and the parafalcine lesion is hypointense. E-F) Unenhanced computed tomography reveals the nature of the quadrigeminal lesion to be a lipoma and the parafalcine lesion to be a calcified meningioma 

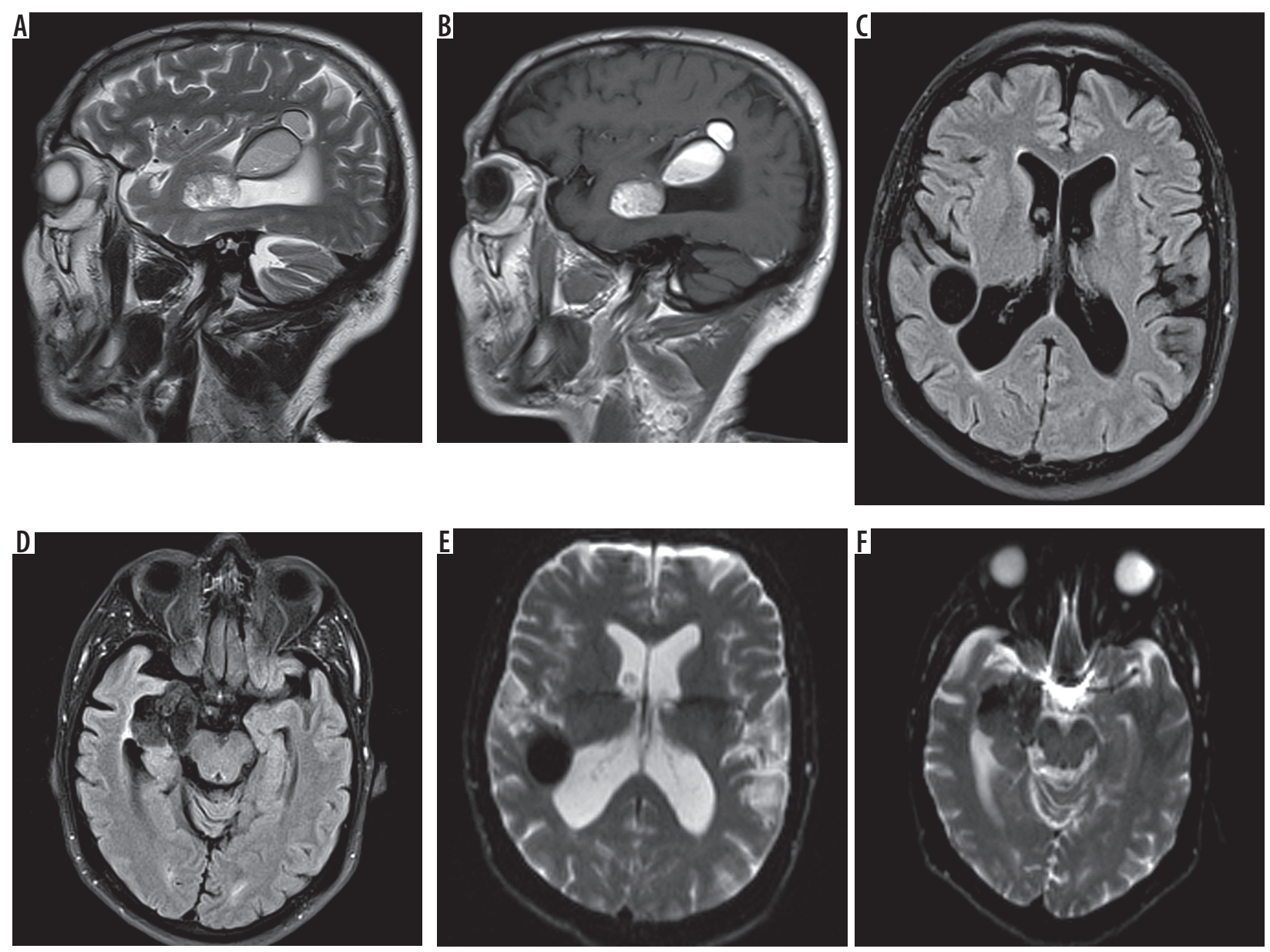

Figure 5. Differentiation of dermoid cysts. A) Sagittal T2-weighted image demonstrating multi-lobulated right temporal dermoid; note the chemical shift artefact and lesion extending superiorly within the parenchyma. B) Sagittal T1-weighted image with the most superior component with homogenous T1 characteristics consistent with predominantly liquid fat material. C) Axial fat suppressed FLAIR image with a homogenously hypointense left posterior temporal lesion; the lesion could easily be incorrectly mistaken for a fluid cyst. E-F) Diffusion-weighted image at B0 demonstrates complete low density with apparent blooming artefact; this could be mistaken for a haemosiderin-containing lesion

\section{References}

1. Muçaj S, Ugurel M, Dedushi K, et al. Role of MRI in Diagnosis of Ruptured Intracranial Dermoid Cyst, Case Report. Acta Inform Med 2017; 25: 141-144.

2. Osborn AG, Preece MT. Intracranial cysts: radiologic-pathologic correlation and imaging approach. Radiology 2006; 239: 650-664.

3. Mehta MP, Chang SM, Guha A, et al. Principles and Practice of Neuro-Oncology: A Multidisciplinary Approach. Demos Medical, 2010.

4. Runge VM, Smoker WRK, Valavanis A. Neuroradiology: The Essentials with MR and CT. Thieme, Berlin 2014.

5. Esquenazi Y, Kerr K, Bhattacharjee MB, et al. Traumatic rupture of an intracranial dermoid cyst: Case report and literature review. Surg Neurol Int 2013; 4: 80.

6. Cohen JE, Abdallah JA, Garrote M. Massive rupture of suprasellar dermoid cyst into ventricles. Case illustration. J Neurosurg 1997; 87: 963.
7. Sood S, Gupta R. Susceptibility artifacts in ruptured intracranial dermoid cysts: a poorly understood but important phenomenon. Neuroradiol J 2014; 27: 677-684.

8. Erasmus LJ, Hurter D, Naude M, et al. A short overview of MRI artifacts. SA J Radiol 2004; 8: 13-17.

9. Zimny A, Zińska L, Bladowska J, Neska-Matuszewska M, Sąsiadek M. Intracranial lesions with high signal intensity on T1-weighted MR images - review of pathologies. Pol J Radiol 2013; 78: 36-46.

10. Tsuruda JS, Chew WM, Moseley ME, et al. Diffusion-weighted MR imaging of the brain: value of differentiating between extraaxial cysts and epidermoid tumors. AJR Am J Roentgenol 1990 11: 925-931. 